vormbgen eingebüfst, jene ist verdünnte Salzsäure, diese reines Pepsin. Analog zerfällt die Holzschwefelsäure unter diesen Verhattmissen in Dextrin und Schwefelstiure.

Die ineisten dieser Thatsachen sind schon von Wassmann und Andern ermittelt; ich bin absiclitlich ihrer Darslellung gefolgt, um bei diesem Versuch einer rationellen theoretischen Begründung des Verdauungsprocesses möglichst unpartheiisch und objectiv zu Werk zu gehen. Er ist auf die bis jetzt ermiltelten Thatsachen gestülyl und erklürt wenigstens das Vorliegende voll Nindigr ans einem einfachen Gesichtspunkl. Ueler die allgemeine Zulässigkieil müssen weilere Versuche enlscheiden.

\title{
Ueber Mikrolirystallomelric;
} von Demselben.

Genauere krystallographische Bestimunungen werden in der organischen Chemie ein läglich fiihlbareres Desiderat. Alonıvolum specifische Wärme, Dichtigkeit, Schmelz- und Siedpunkt versprechen uns die wichtigsten Aufschlüsse über die wahre Constitution der Verbindungen zusammengesetzter Radicale; um wie viel mehr werden uns genaue mathematische Definitionen der Formverhältnisse liefern, deren Kenntnifs und Combination wir bereits die erfolgreiche Lehre des Isomorphismus, die Thatsachen des Di- und Polymorphismus verdanken. Sind die Verbindungen der Aelhyl- und Methylreilıe, die des Amyls, Allyls, Glyceryls u. A., die der Platinbasen, der zahlreichen neuerdings studirten organischen Basen mit denen gewisser Alkaloïde, des Ammoniaks, den Metalloxyden dieser oder jener Gruppe isonorph, oder finden sich bestimute Relationen bei verschiedenen 
Temperaturgraden, verschiedenem Wassergehalt der Krystalle u. s.w. Alle diese Pinkfe sind vom höchsten Interesse; sie sind bei jeder Untersuchungsivii:e gelegentlich leicht experimentell zu erörtern, während die Aufgabe, für sich unternommen, die Ǩräfte des Einzelnen bei weitem ühersteigt - durch gelegentliches Zusammenwirken Aller wird binnen cin Paar Jahren das nöthige Material an Specialbestimmungen zusammengebracht - der Einzelne braucht ein Menschenalter, um nur die Grundsteine zu beschaffen und zu ordnen.

Ein wesentliches Hindernifs bot bisher die mikroscopische Kleinheit oder aufserordenlliche Zersetzbarkeit der gröfseren, hieher gehörigen Krystalle beim Aussetzen an die Luft, oder bei der Befestigung des Krystalls auf der Axe des Reflexionsgoniometers, Manipulation, Erwärmen, Druck der berührenden Hand etc. Es gelang ınir, beide Uebelstände durch eine einfache Messungsund Berechnungsmethode $\mathrm{zu}$ eliminiren, die bei gut gebildeten Krystallindividuen von 1/50 - 1/100"' vollkommen die Sicherheit reflexionsgoniometrischer Bestimmungen gestattet *). In der betreffenden Monographie theilte ich zugleich eine Reihe krystallonomischer Untersuchungen mikroscopischer Krystalle mit, deren Formkenntnifs für die physiologische Chemie, namentlich medicinisch-chemische Diagnostik, von besonderer Wichtigkeit ist. Sie können als Beispiele der Messung und Rechnung dienen; es finden sich Repräsentanten sämnitlicher Krystallsysteme, mit Ausnahme des trillinometrischen (ein- und eingliedrigen) darunter.

Es handelt sich jetzt darum, die Gültigkeit obiger Behauptung in Betreff der Genauigkeit der Messung nach dieser Methode, gegenüber den mit dem Reflexionsgoniometer erhaltenen Resultaten zu begründen. Ich bin in meiner Schrift den Beweis

*) In der schon olsen erwäinten Schrift : „Krystallonomische Uutersuchungen zur physikalischen Chemie". Mitau und leipzig 1846. 8. 
schuldig geblieben - ein glücklicher Zufall erlaubt mir, ihn jetzt mit aller Schärfe zu führen.

Dieser Beweis mufste 1) an Krrystallen gefülurt werden, die sich in der zur reflexion gonionetrischen Messung nöthigen Grörse mit vollkommen ebenen, spiegrehuden Flachen darstellen liefsen; und 2) um jeden Schein von Partheilichkeit zu vermeiden, auf vorhandene, von jedem Vorurtheil unabhängig ausgeführte Messungen mil dem Spiegelgoniometer basirt werden. Dus Resultat dieser letztern mufste mir, um absolul gar nicht zu. Gunsten der mikrogoniomelrischen Messung unwillkürlich während der Ausfïhrung letzterer influiren zu können, vor der Veröllentlichung meiner Untersuchung unbekanut bleiben.

Es ist klar, dafs eine so scrupulöse Controlle der Selbstkritik nur durch ein besonders glückliches Zusammentreffen von Umständen möglich werden komnle; es ist vinfach folgendes :

Unter den Krystallreihen, deren Gesetz ich auf Grund mikrogoniomelrischer Messung festzuslellen gesucht, befand sich das Tripelphosphat. Es war bisher nur in mikroscopischen Formen beobachtet, ich hatte die krystallonomischen Homente an Krystallen von $1 / 30-1 / 200$ " Durchmesser ermittelt.

Das eben angelangte Juniheft des Philosophical Mrgazine nun enthält einen Aufsatz von Teschemach cr über melirere in den Guanolagern und deren Ungebung gufundene Substanzen *).

Am Interessantesten ist für uns das Vorkommen des Tripelphosphats in ausgezeichnel schönen, grofsen Kryslallen, mit treflich spifgelnden, sich für's Ruflexionsgoniometer eignenden Flächen. Sie fanden sich nesterweise in die Guanomasse ein-

*) Nr. LXXXIV. An account of varions substances found in the Guano deposits and in their vicinity. By L. F. Teschemacher Esq. (communicated by the Chemical Snciety; having Jeen read lecumber $I$, 1845.) 
gebettet an der afrikanischen Küste (Saldanha Bay) *). T. schlägt dafiir, da Tripelphosphat bisher nicht fossil gefunden worden, als Mineral den Namen Guanit vor. Grundform ist das gerade rhombische Prisnı, Spaltbarkeit parallel den Seitenflächen desselben, specifisches Gewicht $=1,65$ Härte $=2$ (des Gypses). Die mit dem Reflexionsgoniometer gemessenen Winkel sind:

$$
\begin{aligned}
& M: M^{\prime}=57^{\circ} 30^{\prime} \\
& M: \mathrm{t}=118^{\circ} 30^{\prime} \\
& M^{\prime}: \mathrm{f}=118^{\circ} 30^{\prime} \\
& M^{\prime}: \mathrm{h}=151^{\circ} 00^{\prime} \\
& \mathrm{f}: \mathrm{h}=89^{\circ} 30^{\prime} \\
& M: \mathrm{e}=142^{\circ} 10^{\prime} \\
& M^{\prime}: \mathrm{e}^{\prime}=142^{\circ} 10^{\prime}
\end{aligned}
$$

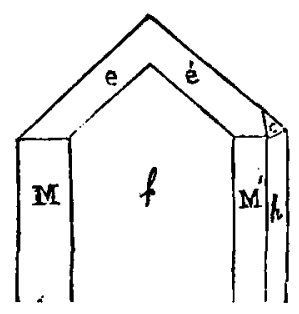

Diese sehr dankenswerthen Beslimmungen theilen den Mangel der zahlreichen Brooke'schen und Phillips'schen Messungen, den nämlich, dafs der Werth der Messungsreihe nicht angegeben und Zahl und voahrscheinlicher Fehler Jer Einzelmessungen nicht bestimmt ist, so dafs es unmöglich ist, nach der Methode der kleinsten Quadrate die vahrscheinlichsten Mittelwerthe zur Bestimmung des Axensystems und der Grundform zu deduciren. Wir sind daher genöthigt, die Messungen als gleichwerthig anzusehen, mithin die letzlerwälsnte Methode (der kleinsten Quadrate) in die des arithmetischen Mitsels übergehın zu lassen.

Aus der Polkantenneigung des Octaëdërs der Grundform $\mathrm{e}: \mathrm{e}^{\prime}=9^{\circ} 50^{\prime}$ und derselben Octaëderflächen gegen den brachydiagonalen Hauptschnitt oder das gleichnamige Flächenpaar $\mathrm{f}-\mathrm{e}: \mathrm{f}=\mathrm{e}^{\prime}: \mathrm{f}=112^{\circ} 20^{\prime}$ ergiebt sich zunächst das

*) l. c. p. 548 : "The third substance was found at Saldanha Bay on the coast of Africa, imbedded in patches in the mass of Guano. It is found in distinct crystals with numerous modifications, many of the planes possessing sufficient brilliancy ta enable me to measure the angles by the reflecting goniometer" elc. 
Krystallsystem als rechtwinklig und zwar als rhombisch (einund einaxig) - ich gelangte (I. c. p. 48) zu dernselben Resultat.

Aus densellen Daten ergiebt sich der Neigungswinkel $\varphi$ des makrodiagonalen Horizontalprismas oder der gleiclinamige Polkuntenwinkel des Rhombenoctaëlers :

$$
\cos 1 / 2 \varphi=\frac{\cos 67^{\circ} 40^{\prime}}{\sin 45^{\circ} 55^{\prime}}=116^{\circ} 8^{\prime}
$$

Der nach der Methode der kleinsten Quadrate aus meinen mikrogoniometrischen Messungen deducirte Werth war $=116^{\circ} 4^{\prime}$

Den Neigungswinkel des Rhombenoctaëders grgen das Prisma der Grundform bestiminte T. $=\mathrm{e}^{\prime}: \mathrm{M}^{\prime}=\mathrm{e}: \mathrm{M}=142^{\circ} 10^{\prime}$; der daraus berechnele Flächenneigungswinkel $\varphi^{\prime}$ des Prismas der Grundform ergiebt sich aus :

$$
\cos 1 / 2 \varphi^{\prime}=\frac{\cos 45^{\circ} 55^{\prime}}{\sin 52^{\circ} 10^{\prime}}=56^{\circ} 31^{\prime}
$$

Derselhe direct gemessen betrug a) $\mathrm{M}: \mathrm{N}^{\prime}=\mathbf{5 7}^{\circ} 30^{\prime}$

wabrscheinlicher Nittelwerth $=\overline{57^{\circ} 0, \overline{5^{\prime}} \text {; }}$ es ergab sich aber derselbe indirect gemessen

b) (aus $M^{\prime}: f=M: f$ )

mithin wahrscheinlicher Werth $\overline{=75^{0} 0,25^{\circ}}$

Meine Messung hatle ergeben $=5^{\circ} 00^{\prime}$

Meine auf Polkantenwinkelınessung basirte Rechnung $=5^{\circ} \mathbf{6}^{\circ}$.

Der Neigungswinkel $\varphi^{\prime \prime}$ des brachydiagonalen Horizontalprismas odcr des gleichnamigen Polkantenwinkels des Rhombenoctaëders ergiebt sich aus T's Messungen (Flächenneigung des Rhombenoctaëders gegen das Prisma der Grundform) e : $f=$ $e^{t}: \mathfrak{f}=67^{\circ} 40^{\prime}$ und Neigungswinkel des letzteren $\mathrm{M}: \mathrm{M}^{\prime}=57^{\circ}$

$\cos 1 / 2 \varphi^{\prime \prime}=\operatorname{tang} 61^{\circ} 30^{\prime} \cdot \operatorname{cotg} 67^{\circ} 40^{\prime}=82^{\circ} 13^{\prime}$.

Meine mikrogoniometrische Messung (1. c. p. 48) ergab ihn $82^{\circ} 10^{\prime}$. Dafs die algeleiteten Winkel dieselbe Uebereinstimmung zeigen müssen, wie diese Grundiverthe, aus denen sie zu deduciren sind, versteht sich von selbst. 
Stellen wir das Resultat schliefslich zusammen :

Flăchenneigung dies Reflexionsgoniometer Mikrogoniometer

Krystall $2-3^{\prime \prime \prime}$ D. Krystall $1 / 30-2 / 100 "$ D.

Prismas der Grundform

$57^{\circ}$

$57^{\circ}$

Makrodiagonaler Polkantenwinkel 116 ${ }^{\circ}$

Brachydiagonaler

$82^{\circ} 13^{\prime}$

$116^{\circ} 4^{\prime}$

Axenverhälınifs a : b : c $0,6233: 1: 0,5429 \quad 0,6241: 1: 0,5441$ Wahrscheinlicher Fehler der

berechneten Winkel 15' $1 \frac{1}{\prime} 2^{\prime}$.

Das Endresultat, dafs bei reflexionsgoniometrischer Messung der wahrscheinliche Fehler zehnfach den aus meinen mikrogoniometrischen Bestimmungen ïbersteigt, mag paradox genug erscheinen - doch, die Thatsachen liegen vor, Jeder kann sich von der Sachlage überzeugen.

Der Grund ist sehr einfach : meine Messungsreihen sind mit ihren wahrscheinlichsten Werthen in die bekannte Gauss'sche Gleichung der kleinsten Fehlerquadrate eingeführt - in denen Teschemacher's mufsten sie sehr willkürlich als gleichwerthig angesehen werden. Bei gleicher Methode der Berechnung fallon die wahrscheinlichen Fehler der Einzelbestimmungen wie die mittleren Fehler ganzer Messungsreilen nach beiden goniometrischen Methoden ziemlich gleich aus; in Betreff des Specielleren mufs ich auf meine ersterwhänle Schrift selbst verweisen *).

*) Auch in Hamburg ist neuerdings beim Aufgraben eines alten Kirchhofs das Tripelphosphat in Folge sehr allmäliger Bildung in ausgezeichnet schönen Krystallen gefunden worden. Ulex, der die Sache (diese Annalen 1846, Januarhefti genauer beschrieben, legt ihnen als Fossil den Namen Struoil bei. Beide Entdeckungen sind gleichzeitig und unhhängig von einander gemacht worden; un etwaigen etymologischen Prioritätsstreitigkeiten a priori mit der gröfston Unpartheilichkeit zu begeguen, wäre es wohl das einfachste, Guanit und Siruvil nach wie vor als Tripelphosphat zu bezeichnen. Der Compendienballast der Synonymik ist grofs genug, und ob ein Jingerhaufen über oder unter der Erde fault, ist in Betreff der blofsen Nomenclatur der Füulnifsproducte sehr gleichgüftig. 